\title{
In-situ Observation of Large Strain Deformation and Failure Behavior of Hydrated Biopolymer Structures Using Environmental Scanning Electron Microscopy
}

\author{
N. Traitler \& A.M. Donald
}

Biological \& Soft Systems, Cavendish Laboratory, Madingley Road, Cambridge, CB3 0HE, UK

One of the most important advantages of environmental scanning electron microscopy (ESEM) is its ability to image hydrated samples [1]. Like conventional scanning electron microscopes, ESEM offers high resolution combined with a good depth of field. Further, the humidity of a sample can be controlled in ESEM by varying the water vapor pressure or temperature [2].

This paper describes a tensometer, as shown in Figure 1, that has been built to fit inside an ESEM, and which is capable of applying large strains to hydrated samples [3-4]. This technique enables us to image the mechanical response of a system, while quantitatively measuring load/extension data. Furthermore, hydrated systems can be observed at room temperature using a novel detection system in the ESEM [5].

In the present study we use the tensometer to investigate large strain deformation and failure behavior of biopolymer cellular solids via in-situ ESEM (Figure 2). Biopolymers are widely used in the food industry, and therefore understanding the influence of composition and environmental parameters on their mechanical response is crucial for achieving desired textural properties.

Samples are prepared from starch-water mixtures heated between two plates, leading to the formation of a cellular structure. The temperature of the plates and time of heating can be controlled to alter sample morphology and properties. Samples were equilibrated inside the ESEM by varying the water vapor pressure from 2 to 15 torr at room temperature, depending on the desired equilibrium relative humidity of the sample. The effect of hydration on the failure mechanism and mechanical response were investigated.

The composition of polymer cellular structures also plays a crucial role on mechanical response. Structural deformation observed in real time in ESEM can be correlated to stress-strain curves obtained, giving information about the elastic modulus and visco-elastic properties of the system. Modulus and tensile strength are very much dependent on the moisture content.

Crack propagation through the material gives significant insight into the mode of fracture. At high moisture contents, ductile fracture dominates, while at lower moisture, the sample will mainly undergo brittle fracture. The difference between these types of fracture is attributed to the extent of plastic deformation. Environmental conditions such as temperature and relative humidity, as well as the composition of the sample, have great impact on crack propagation.

This apparatus, combined with other novel in-situ techniques enables us to investigate the underlying physical concepts governing the changes in mechanical response of hydrated systems. 


\section{References}

[1] A.M. Donald, Nature Materials 2 (2003) 511

[2] F.S. Baker et al., Techniques for structural characterisation of polymers, London; Wiley 2003

[3] D.J. Stokes \& A.M. Donald, J. Mater. Sci. 35 (2000) 599

[4] R. Rizzieri et al., Polymer 44 (2003) 5927

[5] M. Toth \& F.S. Baker, Microsc. Microanal 10 (Suppl 2) (2004) 1062

[6] The author gratefully acknowledges funding from Nestle PTC York and BBSRC and the help of Dr Debbie Stokes

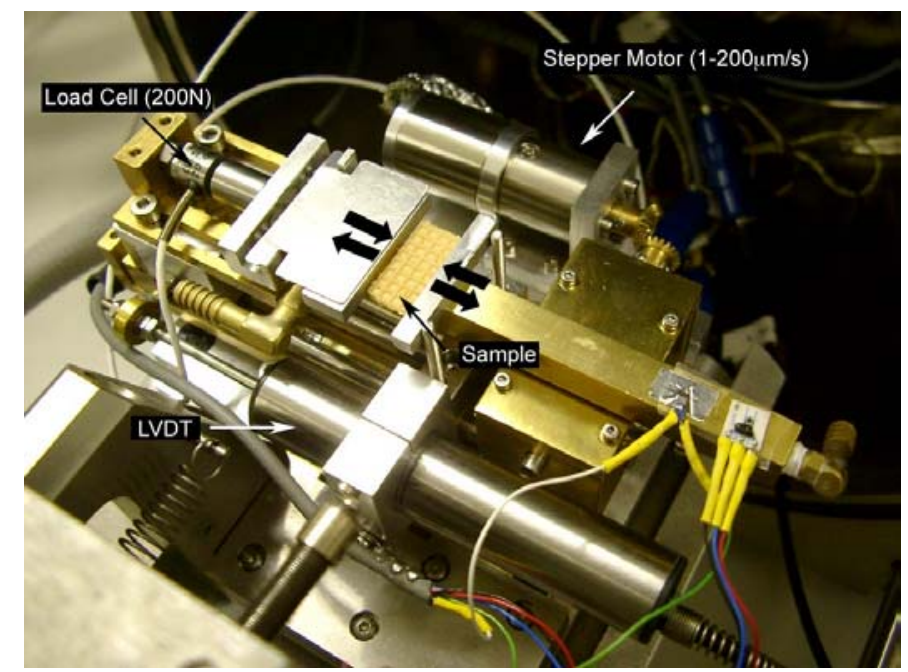

Figure 1 - Photograph of the tensometer, which can be fitted inside the ESEM; the black arrows indicate the direction of compression/tension. The jaws holding the sample are driven by a stepper motor, which controls the displacement rate. A load cell connected to one of the jaws measures the force exerted by the sample as a result of applied load or tensile strain. The temperature of the sample can be controlled by water flow through the block underneath the sample.
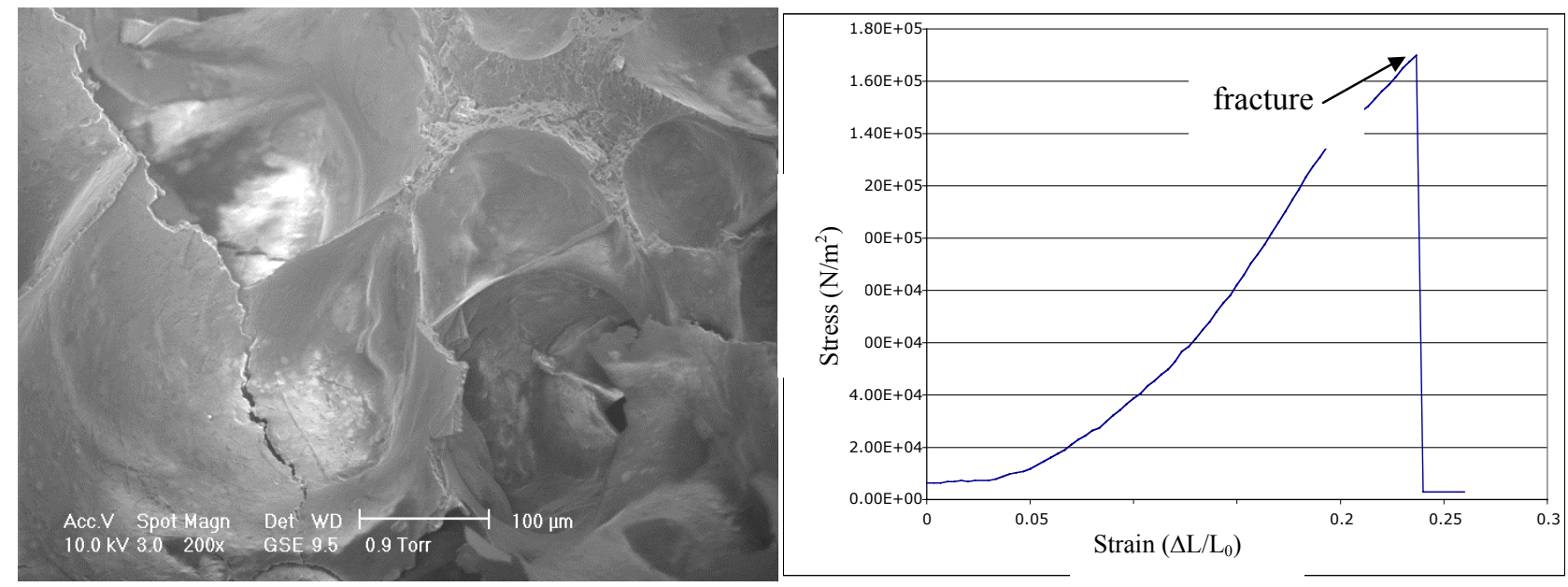

Figure 2 - ESEM micrograph of a crack formation observed after tensile strain of a typical starchbased biopolymer cellular solid. The graph shows the mechanical response of this system as it undergoes tensile strain. 